Abstract

We extend to the lower main sequence stars the analysis of convection interacting with rotation in a compressible spherical shell, already applied to the solar case (Belvedere and Paterno, 1977; Belvedere et al. 1979a). We assume that the coupling constant $\varepsilon$ between convection and rotation, does not depend on the spectral type. Therefore we take $\varepsilon$ determined from the observed differential rotation of the Sun, and compute differential rotation and magnetic cycles for stars ranging from $\mathrm{F} 5$ to $\mathrm{MO}$, namely for those stars which are supposed to possess surface convection zones (Belvedere et al. 1979b, c, d). The results show that the strength of differential rotation decreases from a maximum at F5 down to a minimum at G5 and then increases towards later spectral types. The computations of the magnetic cycles based on the $\alpha \omega$-dynamo theory show that dynamo instability decreases from F5 to G5, and then increases towards the later spectral types reaching a maximum at mo. The period of the magnetic cycles increases from a few years at F5 to about 100 years at MO. Also the extension of the surface magnetic activity increases substantially towards the later spectral types. The results are discussed in the framework of wilson's (1978) observations.

\title{
References
}

Belvedere, G. and Paterno, L. 1977 Solar Phys. 54, 289.

Belvedere, G., Paterno, L. and Stix, M. 1979 a Geophys. Astrophys. Fluid Dyn. (in press). Belvedere, G., Patemo, L. and Stix, M. 1979b Astron. Astrophys. submitted.

Belvedere, G., Paterno, L. and S.tix, M. 1979c Astron. Astrophys. submitted.

Belvedere, G., Paterno, L. and Stix, M. 1979d Astron. Astrophys. to be submitted. wilson, O.C. 1978, Astrophys. J. 226, 379.

\section{CHANGES OF PHOTOSPHERIC LINE ASYMMETRIES WITH EFFECTIVE TEMPERATURE}

David F. Gray

Department of Astronomy, University of western Ontario

London, Canada

Abstract

Asymetries in photospheric lines were described briefly in my earlier contribution to these proceedings and in more detail in the January 15, 1980 issue of the Ap. J. The cores of the stronger lines in $\alpha$ Boo (K2III) show a blue shift, quite opposite to the effect seen in the solar lines. Suspecting this difference to be due to the 
shift in ionization with effective temperature, two other stars were measured, one slightly hotter than $\alpha$ Boo ( $\beta$ Gem KOIII) and one slightly cooler ( $\beta$ UMi K4IV). The preliminary results seem to confirm the effective temperature dependence since no asymmetries were found for $\beta$ Gem and stronger blue-shifted core asymmetries were found for $\beta$ UMi.

\section{SMALL-SCALE VERSUS LARGE SCALE MOTIONS IN THE SOLAR ATMOSPHERE DERIVED FROM}

\section{A NON-ITE CALCULATION OF MULTIPIET 38 OF TI I}

\section{R. Cayrel}

CFHT Corporation, Waimea office

Kamuela HA 96743, U.S.A.

S. Dumont and P. Martin

Observatoire de Meudon

F-92190 Meudon, France

Abstract

A non-LTE computation of multiplet 38 of $T i$ I (1I lines) has been undertaken in order to determine small and large scale unresolved motions contributing to the Doppler broadening of solar lines at the centre of the disk (vertical motions) and at the edge of the disk (horizontal motions).

The abundance of Titanium and the total Doppler velocity (all scales) axe determined by fitting observed and computed profiles of weak unsaturated lines $(W<12 \mathrm{~m} \AA)$ of the multiplet. Then saturated lines having the same lower level as the weak lines are computed for a variety of partitions of the total kinetic energy between the small scale and the large scale modes going from $0 \%$ small scale to $100 \%$ small scale. Oscillator strengths with internal accuracies of about 28 from wahling (1977) have been used. The location of the observed profile (taken from Delbouille et al. for the centre of the disk and from Brault and Testerman, kPNo for $\mu=0.2$ ) among the computed profiles yields the partition of the energy between the two modes.

The computations done so far with 4 levels and continuum give less than $20 \%$ of the energy in the small scale mode. Further computations with more levels are needed to establish this ratio with better accuracy. The total energy for vertical motions has a root square velocity of about $1.4 \mathrm{~km} / \mathrm{sec}$ whereas the same quantity is $2.2 \mathrm{~km} / \mathrm{sec}$ for horizontal motions. 